\title{
Características clínicas, radiológicas y auxológicas de pacientes con displasia cleidocraneal seguidos en un hospital pediátrico de referencia en Argentina Clinical, radiological, and auxological characteristics of patients with cleidocranial dysplasia followed in a pediatric referral hospital in Argentina
}

\section{Dra. Rosario Ramos Mejía ${ }^{a}$ Dra. Mercedes Rodríguez Celin ${ }^{a}$ y Dra. Virginia Fano ${ }^{a}$}

\section{RESUMEN}

La displasia cleidocraneal es una displasia esquelética autosómica dominante causada por mutaciones en el gen RUNX2, con una prevalencia estimada de $1 / 1000000$ de recién nacidos. Se presentan 37 pacientes (22 mujeres) evaluados entre 1992 y 2016 en las clínicas de displasias esqueléticas, Hospital Garrahan, Argentina. Hallazgos: $35 \%$ de antecedentes familiares positivos; edad mediana al momento del diagnóstico: 2,61 años; características radiológicas positivas en el cráneo y el pubis: 95\%; en las clavículas: $100 \%$. Las complicaciones dentales y auditivas fueron comunes. Auxología: mediana de estatura de $-1,81(-3,26-0,2)$ DE en los varones, $-1,36(-4,28-1,36) \mathrm{DE}$ en las mujeres. Cinco de trece pacientes fueron bajos para la estatura parental. Estatura adulta (mediana): $162,8 \mathrm{~cm}$ y $149,2 \mathrm{~cm}$ en los varones y las mujeres. Nofueron evidentes alteraciones en la proporción estatura sentada / estatura. Un paciente presentó macrocefalia real; $12(32 \%)$, macrocefalia relativa. Se describe variabilidad intrafamiliar de estatura. Palabras clave: displasia cleidocraneal, crecimiento, clavicula, fontanelas craneales.

http:/ / dx.doi.org/10.5546/ aap.2018.e560 Texto completo en inglés:

http:/ / dx.doi.org/10.5546/ aap.2018.eng.e560

a. Clínicas

Multidisciplinarias de Displasias Esqueléticas, Hospital Garrahan, Ciudad Autónoma de Buenos Aires,

Argentina.

Correspondencia:

Dra. Rosario Ramos Mejía: rosariorm@gmail.com.

Financiamiento:

Ninguno

Conflicto de intereses: Ninguno que declarar.

Recibido: 6-9-2017 Aceptado: 22-1-2018
Cómo citar: Ramos Mejía R, Rodríguez Celin M, Fano V. Características clínicas, radiológicas y auxológicas de pacientes con displasia cleidocraneal seguidos en un hospital pediátrico de referencia en Argentina. Arch Argent Pediatr 2018;116(4):e560-e566.

Cbfa1: core-binding factor-1 (factor 1

de unión al núcleo).

CDC: Centro de Control de

Enfermedades.

DCC: displasia cleidocraneal.

DE: puntaje del desvío estándar.

IMC: índice de masa corporal.

\section{GLOSARIO}

OMS: Organización Mundial de la Salud.

RCIU: retraso de crecimiento intrauterino.

RUNX2: runt-related transcriptor factor 2 (factor de transcripción relacionado con runt 2).

TNSALP: fosfatasa alcalina no específica de tejido.

\section{INTRODUCCIÓN}

La displasia cleidocraneal (DCC) (MIM 119600) es una displasia esquelética caracterizada por fontanelas permeables y aplasia o hipoplasia clavicular, con amplia variabilidad fenotípica. ${ }^{1,2}$ Se describe que la población afectada puede presentar baja estatura moderada, ${ }^{1,3-5}$ pero se han reportado pocos datos acerca de otras variables auxológicas.

Su prevalencia es de 1 en 1000000 de nacimientos y es independiente del sexo. ${ }^{4}$

La DCC es causada por mutaciones, heredadas de manera autosómica dominante, en el gen RUNX2, en el brazo corto del cromosoma $6.1,2,4,6$ Se han identificado inserciones, deleciones, mutaciones de cambio de sentido y mutaciones sin sentido. ${ }^{6-10}$ En la mayoría de los casos, estas mutaciones son de novo. ${ }^{4}$ Se ha propuesto también mosaicismo gonadal. ${ }^{1}$ Las variantes patógenas se han encontrado en el 60-70\% de los individuos con diagnóstico clínico. ${ }^{4}$

El gen RUNX2 codifica para el factor de transcripción Cbfa1, esencial para la diferenciación de osteoblastos y la morfogénesis del esqueleto, con 
participación en la osificación intramembranosa y endocondral. Las mutaciones en RUNX2 resultan en un trastorno que combina defectos aislados de hueso (disostosis) con defectos progresivos de tejidos (displasia). ${ }^{2}$

Se ha descrito un pequeño subgrupo de pacientes con características clínicas y de laboratorio que se solapan con la hipofosfatasia, posiblemente debido a una alteración en la expresión del gen TNSALP (fosfatasa alcalina no específica de tejido). 2,5,11,12 Se ha demostrado que los ratones knockout Runx2 exhiben una falta completa de la mineralización y ausencia de expresión de TNSALP. ${ }^{13}$

El diagnóstico de la DCC se basa en hallazgos clínicos y radiológicos..$^{1,2,4}$ Dentro de los diagnósticos diferenciales, deben incluirse el hipotiroidismo, la pseudoartrosis congénita de clavícula (MIM 118980) y otras displasias esqueléticas. ${ }^{1,4}$

Se ha recomendado un seguimiento en búsqueda de complicaciones ortopédicas, dentales y de la audición, infecciones de los senos paranasales y del oído medio, obstrucción de la vía aérea superior y osteoporosis., ${ }^{3,4}$ Si bien algunos autores han informado retraso en la adquisición de pautas motoras, ${ }^{3,4}$ se describe que la escolaridad, la esperanza de vida y la funcionalidad son normales. ${ }^{2,3}$

\section{OBJETIVOS}

Descripción clínica y auxológica de pacientes con diagnóstico de DCC.

\section{MATERIALES Y MÉTODOS}

Se llevó a cabo una revisión de los registros médicos de pacientes con diagnóstico clínico y radiológico de DCC. Este estudio retrospectivo se basó en los datos de los niños y sus padres evaluados entre 1992 y 2016, en las clínicas multidisciplinarias de displasias esqueléticas en el Hospital "Prof. Dr. Juan P. Garrahan", Argentina.

Se consideraron las siguientes variables: edad, edad al momento del diagnóstico, sexo, motivo de consulta, miembros de la familia afectados, peso, longitud corporal, estatura, estatura sentado, perímetro cefálico y la presencia de complicaciones asociadas.

Las mediciones antropométricas se llevaron a cabo por el mismo observador en el laboratorio de antropometría del Servicio de Crecimiento y Desarrollo, empleando técnicas estandarizadas recomendadas por la Sociedad Argentina de Pediatría. ${ }^{14}$
Se usaron instrumentos Harpenden para la medición de la longitud corporal, la longitud vértex-nalga, la estatura y la estatura sentado. Se utilizó una balanza de palanca para el peso. Los errores de medición intraobservador fueron $0,11 \mathrm{~cm}, 0,10 \mathrm{~cm} \mathrm{y} 0,10 \mathrm{~kg}$ para la longitud corporal, la estatura y el peso, respectivamente.

El peso y la longitud corporal al nacer se obtuvieron de los registros neonatales de los pacientes cuando estuvieron disponibles.

Los familiares adultos afectados se consideraron para el análisis de la estatura final.

Se calcularon puntajes del desvío estándar (DE) para los datos antropométricos. La estatura y las proporciones corporales (estatura sentado/ estatura y perímetro cefálico/estatura) se compararon con estándares argentinos; ${ }^{15-16}$ datos británicos de 1990 se utilizaron para evaluar el perímetro cefálico. ${ }^{17}$ Los datos de índice de masa corporal (IMC) se compararon con datos del estudio multicéntrico de la Organización Mundial de la Salud (OMS) en niños menores de 5 años y con datos del Centro de Control de Enfermedades (Centers for Disease Control and Prevention, CDC) en niños mayores de 5 años de edad. ${ }^{18,19}$

Se calculó el promedio de la estatura parental (midparental height; $\mathrm{MPH}$, por sus siglas en inglés) en los casos en los que ambos padres no estuvieron afectados, expresados como $\mathrm{DE}: \mathrm{MPH}=(\mathrm{PZP}+\mathrm{PZM}) / 2$, donde PZP es el puntaje $\mathrm{Z}$ de estatura del padre y PZM es el puntaje $\mathrm{z}$ de estatura de la madre, considerando $\mathrm{MPH} \pm 1,28 \mathrm{DE}$ como el rango genético normal en niños prepúberes. ${ }^{20}$

Se consideró que los pacientes habían llegado a su estatura final cuando hubieron alcanzado el desarrollo puberal adulto (estadio 5 de mamas o dos años después de la menarca en las mujeres; volumen testicular de $15 \mathrm{ml}$ en los varones).

El diagnóstico radiológico fue confirmado por dos de los autores (MRC, RRM). Los criterios diagnósticos para cada característica radiológica fueron los siguientes: presencia de huesos wormianos cuando la frecuencia de aparición fue 10 o más, ${ }^{21}$ cierre retardado de la fontanela anterior cuando se mantuvo abierta después de los primeros 2 años de vida, ${ }^{22}$ clavículas hipoplásicas cuando estaba ausente la mitad lateral de la clavícula, ${ }^{22}$ retraso en la osificación de los huesos del pubis cuando no estaban osificados más allá de los primeros 6 meses de vida y falta de fusión de ramas isquiopubianas cuando no existía fusión en los pacientes de 12 años y mayores, ${ }^{22-24}$ anomalías en las manos cuando había retraso en 
la osificación de los huesos del carpo o existían epífisis en cono o seudoepífisis. ${ }^{2}$

Las complicaciones se consideraron cuando estuvieron registradas en la historia clínica.

\section{RESULTADOS}

El estudio incluyó 37 casos con DCC: 59\% de niñas $(n=22)$. La mediana de edad fue 1,59 (de 0 a 16,09) años en la primera visita y 2,61 (de 0 a 16,09) años al momento del diagnóstico. Los pacientes tuvieron seguimiento durante una mediana de 3,67 (de 0 a 12,67) años. En el 35\% de los casos $(n=13)$, se encontraron miembros de la familia afectados.

Las dos razones principales de consulta fueron anomalías del cráneo (asimetría de cráneo, fontanela amplia, diástasis de suturas) en el 51\% $(\mathrm{n}=19)$ y sospecha de displasia esquelética en el $19 \%$ ( $n=7,2$ de ellos con sospecha de DCC). Otras razones fueron coxa vara o valga $(n=6)$, baja estatura $(n=4)$, escoliosis $(n=2)$, alteraciones en las clavículas $(n=2)$, hipotonía $(n=1)$ y retraso en la adquisición de pautas motoras $(n=1)$. Algunos pacientes tenían más de un motivo de derivación.

La Tabla 1 muestra los hallazgos clínicos; los datos se comparan con la literatura.

\section{Análisis auxológico}

Niños $(n=15)$

Se contaba con datos de antropometría al nacer en cuatro casos. Un paciente presentó restricción del crecimiento intrauterino (RCIU) en peso, longitud corporal y perímetro cefálico sin razón atribuible.

La Figura 1. A muestra la curva de distancia de estatura en los varones; es evidente que todos, excepto uno, tienen un crecimiento normal. Este paciente está siendo estudiado por retraso del crecimiento; tiene parámetros de laboratorio normales. La mediana de estatura en la última visita fue $-1,81$ (de $-3,26$ a 0,2 ) DE y la mediana de edad, 6,43 (de 0,92 a 16,79) años. Para los pacientes con datos de estatura de padres reportados $(n=8)$, la mediana de las diferencias entre $\mathrm{MPH}$ en DE y la estatura de los niños en DE fue -1,12 (de -2,03 a $1,44) \mathrm{DE}$; tres pacientes eran bajos y uno alto para la estatura de los padres.

La mediana del perímetro cefálico en la última visita fue $-0,85$ (de $-2,23$ a 0,81 ) DE. Ningún niño tuvo verdadera macrocefalia; sin embargo, todos menos uno $(n=14)$ se encontraban por encima del centilo 50 en la curva de perímetro cefálico.

TABLA 1. Características clínicas en la muestra de 37 pacientes. Hallazgos reportados en la literatura ${ }^{3,25}$

\begin{tabular}{|c|c|c|c|}
\hline \multicolumn{2}{|c|}{ Características clínicas } & \multirow{2}{*}{$\begin{array}{c}\text { Casos }(\mathbf{n}=37) \\
\% \\
73\end{array}$} & \multirow{3}{*}{$\begin{array}{c}\text { Cooper }(n=283), \\
\text { Golan }(n=90) \\
\% \\
98\end{array}$} \\
\hline Dentomaxilar & $\begin{array}{l}\text { Retraso en la dentición primaria } \\
\text { y secundaria, dientes hipoplásicos, } \\
\text { defectos del esmalte }\end{array}$ & & \\
\hline & Paladar ojival & 8 & \\
\hline & Fisura del paladar blando & 3 & 4,4 \\
\hline \multirow[t]{2}{*}{ Audición } & $\begin{array}{l}\text { Audiometría u otoemisiones } \\
\text { acústicas patológicas }\end{array}$ & 30 & 38 \\
\hline & Retraso en el lenguaje & 8 & \\
\hline \multirow[t]{3}{*}{ Vía aérea superior } & Otitis media crónica & 14 & 62 \\
\hline & Sinusopatía crónica ${ }^{34,4}$ & 11 & 34,4 \\
\hline & Roncopatía/adenoamigdalectomía & 11 & 8 \\
\hline Respiratorio & Broncoespasmos recurrentes & 19 & 11 \\
\hline \multirow[t]{3}{*}{ Ortopedia } & $\begin{array}{l}\text { Anomalía de cadera } \\
\text { ( } 8 \text { coxa vara, } 1 \text { coxa valga) }\end{array}$ & 24 & \\
\hline & Escoliosis & 16 & 17 \\
\hline & $\begin{array}{l}\text { Deformidad de pectus } \\
\text { (6 excavatum, } 1 \text { carinatum) }\end{array}$ & 19 & \\
\hline Escolaridad & & normal & \\
\hline
\end{tabular}


Como se muestra en la Figura 2.A, siete pacientes presentaron macrocefalia relativa.

Los pacientes no mostraron alteraciones en la proporción estatura sentada/estatura.

El IMC en la última visita fue $-0,36$ (de $-2,41$ a 1,19$)$ DE. Un paciente era delgado y uno tenía sobrepeso.

\section{Niñas $(n=22)$}

Los datos de antropometría al nacer estuvieron disponibles en la mitad de los pacientes $(n=11)$. Solo una niña tuvo RCIU en longitud corporal y perímetro cefálico, sin una razón atribuible.

La Figura 1.B muestra la curva de distancia de estatura en las mujeres; la mediana de estatura en la última visita fue $-1,36$ (de $-4,28$ a 1,36) DE y la mediana de edad, 5,70 (de 0,44 a 19,0 años). Una niña tenía extremada baja estatura, con valores de laboratorio normales. Para las pacientes con

Figura 1 (a y b). Curvas de estatura de niños y niñas, respectivamente

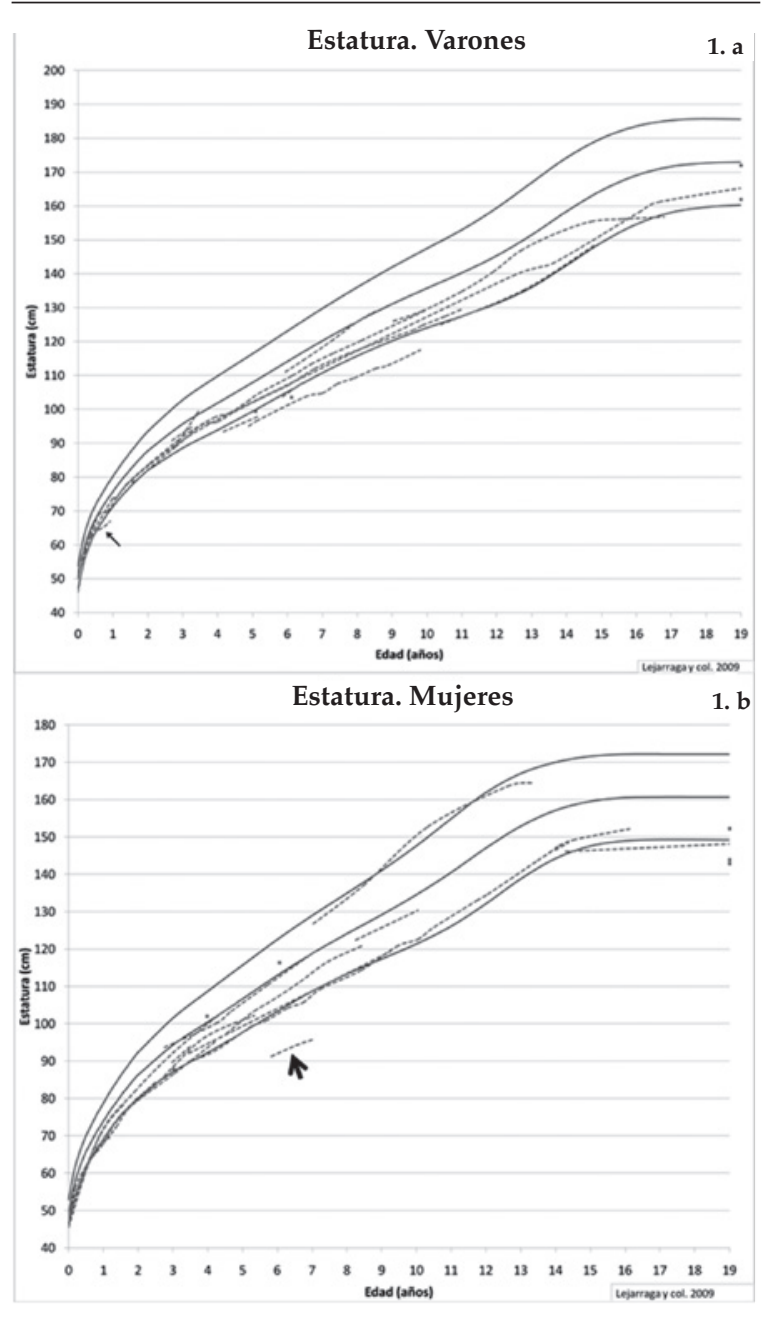

datos de estatura de padres reportados $(n=5)$, la mediana de las diferencias entre $\mathrm{MPH}$ en $\mathrm{DE}$ y la altura de las niñas en DE fue - 0,88 (de $-2,88$ a 0,78$)$ DE. Dos pacientes fueron bajas para la estatura de los padres.

El perímetro cefálico en la última visita fue $-0,04$ (de $-3,47$ a 2,4) DE. La mayoría de las niñas $(n=19)$ se encontraban por encima del centilo 50 en la curva de perímetro cefálico; una de ellas tenía macrocefalia real. El gráfico de perímetro cefálico/estatura (Figura 2.B) muestra que cinco niñas tenían macrocefalia relativa.

No hubo alteraciones en la proporción estatura sentada/estatura.

El IMC en la última visita fue 0,23 (de $-1,46$ a 1,6) DE. Una niña tenía sobrepeso.

\section{Estatura final}

En cuatro varones, estuvieron disponibles los datos de estatura adulta (dos pacientes que alcanzaron la madurez y dos padres afectados); la mediana de estatura fue $162,8 \mathrm{~cm}$, que correspondía a $-1,47$ (de -2,41 a -0,67) DE (Figura 1.A).

FIgURA 2 (a y b). Curvas de perímetro cefálico/estatura para niños y niñas, respectivamente

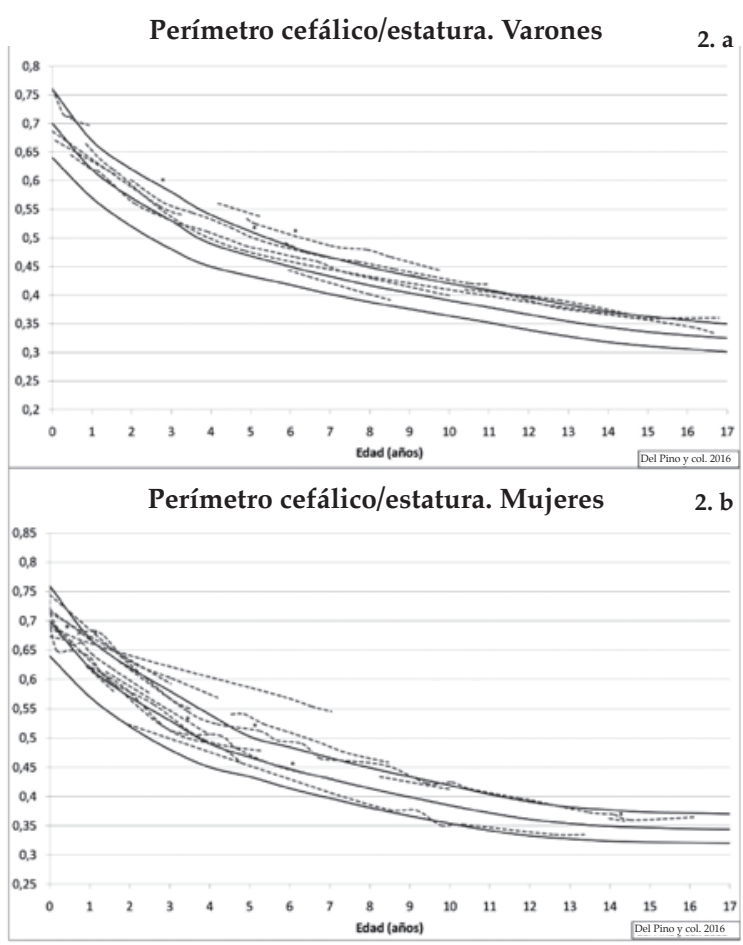


En las mujeres, los datos de estatura adulta estuvieron disponibles en siete casos (cuatro pacientes que alcanzaron la madurez y tres madres afectadas). La mediana de estatura fue $149,2 \mathrm{~cm}$, correspondiente a $-1,89$ (de $-3,45$ a 0,62) DE (Figura 1.B).

Aunque los datos eran pocos, al comparar los de estatura entre los padres afectados y sus descendientes, parecía existir variabilidad intrafamiliar. En 5/10 casos, se encontraron diferencias entre la estatura del padre afectado y la estatura de su descendencia de más de $1 \mathrm{DE}$ (Tabla 2). En una familia con seis individuos afectados, se observó variabilidad de estatura entre -0,74 y -2,05 DE (Figura 3).

\section{Hallazgos radiológicos}

Las características radiológicas de DCC fueron positivas en una alta proporción de las radiografías analizadas: anomalías de cráneo en $95 \%$, alteraciones en las clavículas en $100 \%$ (afectación bilateral en $75 \%$ ), retraso en la osificación de los huesos del pubis en $96 \%$ y alteraciones en las manos en $100 \%$ de los casos (Figura 4, Imágenes 1, 2, 3 y 4).

\section{DISCUSIÓN}

Nuestro estudio muestra una descripción longitudinal de características auxológicas de una serie de pacientes con DCC. De un total de 1409 pacientes evaluados entre 1992 y 2016 en las clínicas multidisciplinarias de displasias esqueléticas del Hospital Garrahan, 37 (2,7\%) han tenido diagnóstico de DCC.
La mediana de edad al momento del diagnóstico fue 2,61 años. Ninguno de los pacientes tuvo diagnóstico prenatal de DCC, aunque algunos tuvieran antecedentes familiares de esta enfermedad. Esto coincide con la literatura, en la que se reconoce que el diagnóstico precoz de la DCC puede ser difícil debido a las manifestaciones frecuentemente leves y a que existe amplia variabilidad fenotípica. ${ }^{2}$

FIGURA 3. Variabilidad intrafamiliar en estatura en un caso

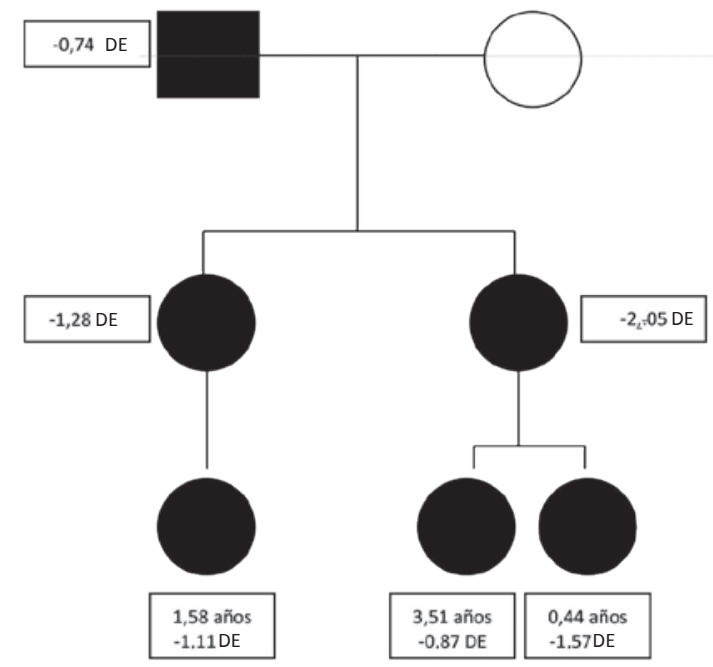

DE: puntaje del desvío estándar.

TABLA 2. Datos comparativos de estatura entre padres afectados y sus descendientes, y entre hermanos afectados

\begin{tabular}{lcccc} 
Caso & Estatura del padre afectado (DE) & Edad del paciente (años) & Estatura del paciente (cm) & Estatura del paciente (DE) \\
\hline 1 & $-1,64$ & 7,04 & 95,80 & $-4,28^{*}$ \\
2 & $-0,74$ & 19,00 & 152,90 & $-1,28$ \\
3 & $-0,74$ & 19,00 & 148,20 & $-2,05^{*}$ \\
4 & $-2,05$ & 3,51 & 94,00 & $-0,87^{*}$ \\
5 & $-2,05$ & 0,44 & 61,00 & $-1,57$ \\
6 & $-1,28$ & 1,58 & 78,40 & $-1,11$ \\
7 & $-2,76$ & 14,36 & 148,50 & $-1,46^{*}$ \\
8 & $-1,40$ & 6,43 & 107,80 & $-1,85$ \\
9 & $-2,94$ & 0,92 & 67,00 & $-3,26$ \\
10 & $-1,98$ & 3,45 & 99,40 & $0,20^{*}$ \\
\hline
\end{tabular}

DE: puntaje del desvío estándar.

Los casos 2, 3, 4, 5 y 6 pertenecen a la misma familia. Los casos 2 y 3 son hermanos. Los casos 4 y 5 son hermanos, descendientes del caso 3. El caso 6 es descendiente del caso 2.

En 5/10 casos, marcados con asterisco $\left(^{*}\right)$, se encontraron diferencias entre la estatura del padre afectado y la estatura de su descendencia de más de 1 DE. Nótense las diferencias entre hermanos afectados. 
Similar a lo mencionado en la bibliografía, las anomalías dentales, los trastornos de la vía aérea superior y de la audición fueron frecuentes. . $^{1,3,4,25}$ Otras anomalías esqueléticas descritas, como retraso en la osificación de los huesos del pubis, coxa vara o valga, escoliosis, deformidad de pectus, también fueron encontradas en este grupo de pacientes. ${ }^{3,4}$

En cuanto al desarrollo intelectual, en nuestro grupo, así como lo descrito por Cooper, no se han encontrado diferencias significativas en cuanto al nivel de estudios alcanzados entre los individuos afectados y sus pares no afectados. ${ }^{3}$

Esta condición no se conoce como una condición de baja estatura extrema. ${ }^{3}$ Respecto a los datos de crecimiento, solo dos pacientes tuvieron RCIU, sin otra causa conocida. A pesar del tamaño pequeño de la muestra (once adultos), la estatura final fue cercana a los valores informados en la literatura; en los varones, la mediana fue $162,8 \mathrm{~cm}$ y, en las mujeres, fue $149,2 \mathrm{~cm}$, mientras que la literatura refiere $165,0 \mathrm{~cm}$ y $156,0 \mathrm{~cm}$ en varones y mujeres, respectivamente. ${ }^{3}$
Se ha encontrado que algunos de los pacientes fueron bajos para el rango genético de estatura cuando los padres no estaban afectados. Además, hubo un niño con retraso de crecimiento y una niña con extrema baja estatura (-4,28 DE), casos que no han sido reportados en la literatura. En el caso de la niña, no se encontró otra causa de baja estatura; su padre estaba afectado, pero sin estatura extremadamente baja $(162,0$ cm, -1,64 DE), lo que mostró variabilidad intrafamiliar.

Con respecto al perímetro cefálico, solo un paciente tuvo verdadera macrocefalia, mientras que el $32,4 \%$ de los pacientes tenía macrocefalia relativa.

No existió alteración en las proporciones corporales evaluadas como la relación estatura sentado/estatura cuando se compararon con los datos argentinos. ${ }^{16}$

Nuestras interpretaciones son limitadas debido a la ausencia de análisis molecular, que, en este grupo, podría mostrar variaciones moleculares.
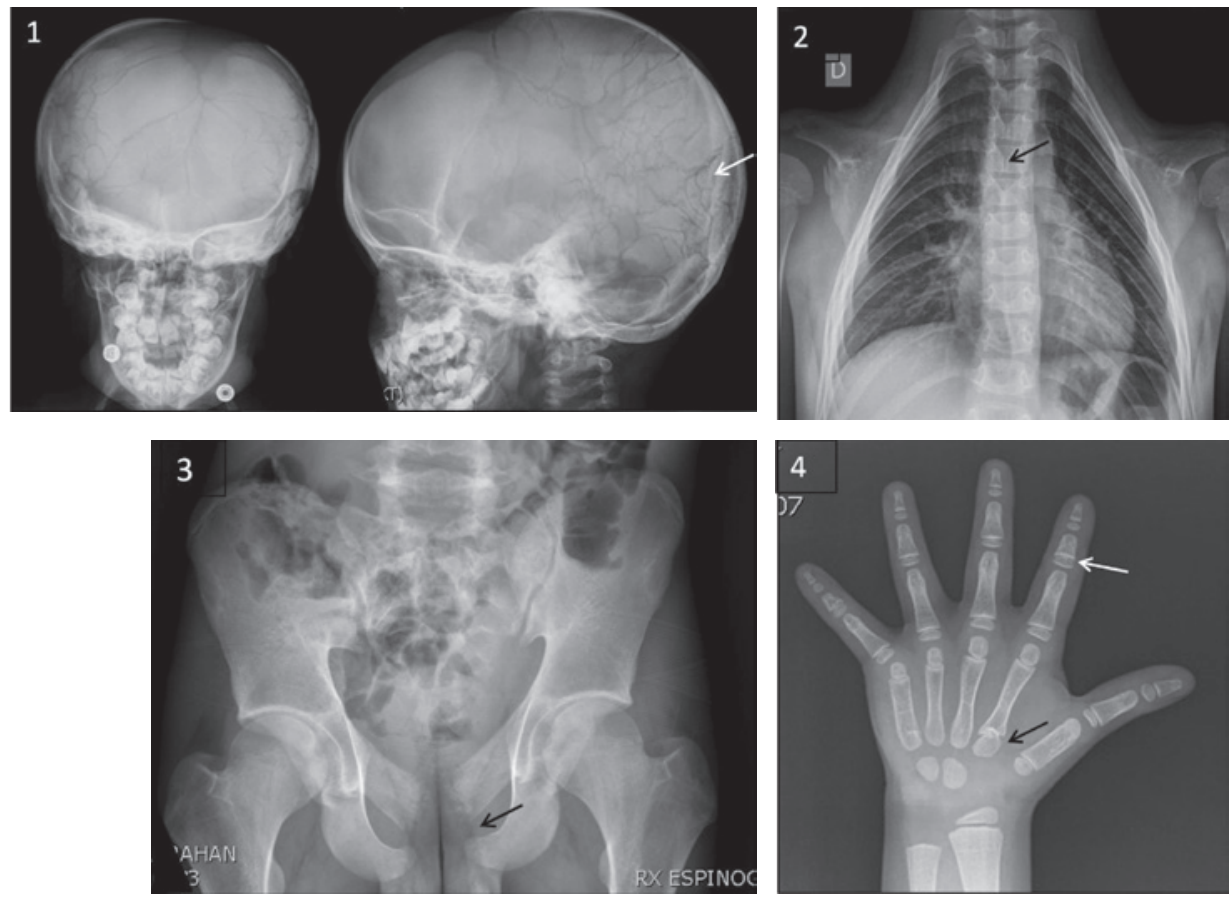

Imagen 1: cráneo, incidencias de frente y lateral. Niña, 5,9 años. Fontanela anterior permeable, huesos wormianos (flecha delgada blanca), hipoplasia del medio facial.

Imagen 2: tórax, frente. Niño, 11,7 años. Ausencia bilateral de clavículas. Disrafismo espinal (flecha delgada)

Imagen 3: pelvis, frente. Varón adulto. Falta de fusión de las ramas isquiopubianas (flecha delgada)

Imagen 4: mano izquierda, frente. Niña, 6,0 años. Retraso en la osificación carpal. Seudoepífisis en metacarpianos (flecha delgada). Epífisis en cono (flecha delgada blanca). 


\section{CONCLUSIÓN}

La mediana de estatura en los pacientes con DCC se encontró dentro de los límites normales; sin embargo, tanto en los varones como en las mujeres, estuvo por debajo del centilo $10 \mathrm{y}$ algunos de los pacientes tenían baja estatura leve. Con respecto a las proporciones corporales, la mayoría de los pacientes no tenían alteraciones, pero un tercio mostró macrocefalia relativa. Aunque estaban disponibles pocos datos sobre familiares afectados, se encontró variabilidad intrafamiliar de altura.

\section{REFERENCIAS}

1. Online Mendelian Inheritance in Man (OMIM $\left.{ }^{\circledR}\right)$. An online catalog of human genes and genetic disorders. Baltimore (MD): Johns Hopkins University; 1966. [Consulta: 17 de diciembre de 2016]. Disponible en: http:/ / omim.org/.

2. Spranger J, Brill P, Nishimura G, et al. Bone Dysplasias: an atlas of genetic disorders of skeletal development. 3rd ed. Oxford: Oxford University; 2012.

3. CooperSC, Flaitz CM, Johnston DA, et al. A Natural History of Cleidocranial Dysplasia. Am J Med Genet. 2001;104(1):16.

4. Machol K, Mendoza-Londono R, Lee B. Cleidocranial Dysplasia Spectrum Disorder. In Adam MP, Ardinger HH, Pagon RA, et al, eds. GeneReviews®. Seatle; University of Washington; 1993-2018.

5. Unger S, Mornet E, Mundlos S, et al. Severe cleidocranial dysplasia can mimic hypophosphatasia. Eur J Pediatr. 2002;161(11):623-6.

6. Mundlos S. Cleidocranial dysplasia: clinical and molecular genetics. J Med Genet. 1999;36(3):177-82.

7. Lee B, Thirunavukkarasu K, Zhou L, et al. Missense mutations abolishing DNA binding of the osteoblastspecific transcription factor OSF2 / CBFA1 in cleidocranial dysplasia. Nat Genet. 1997;16(3):307-10.

8. Quack I, Vonderstrass B, Stock M, et al. Mutation analysis of core binding factor A1 in patients with cleidocranial dysplasia. Am J Hum Genet. 1999;65(5):1268-78.

9. Guo YW, Chiu CY, Liu CL, et al. Novel mutation of RUNX2 gene in a patient with cleidocranial dysplasia. Int J Clin Exp Pathol. 2015;8(1):1057-62.

10. Zhou G, Chen Y, Zhou L, et al. CBFA1 mutation analysis and functional correlation with phenotypic variability in cleidocranial dysplasia. Hum Mol Genet. 1999;8(12):2311-6.

11. El-Gharbawy AH, Peeden JN Jr, Lachman RS, et al. Severe Cleidocranial dysplasia and Hypophosphatasia in a child with microdeletion of the C-terminal region of RUNX2. Am J Med Genet A. 2010;152A(1):169-74.

12. Morava E, Kárteszi J, Weisenbach J, et al. Cleidocranial dysplasia with decreased bone density and biochemical findings of hypophosphatasia. Eur J Pediatr. 2002;161(11):619-22.

13. Komori T, Yagi H, Nomura S, et al. Targeted Disruption of Cbfa1 Results in a Complete Lack of Bone Formation owing to Maturational Arrest of Osteoblasts. Cell. 1997;89(5):755-64.

14. Comité Nacional de Crecimiento y Desarrollo. Guía para la evaluación del crecimiento físico. Buenos Aires: Sociedad Argentina de Pediatría; 2013.

15. Lejarraga H, del Pino M, Fano V, et al. Referencias de peso y estatura desde el nacimiento hasta la madurez para niñas y niños argentinos: Incorporación de datos de la OMS de 0 a 2 años, recálculo de percentilos para obtención de valores LMS. Arch Argent Pediatr. 2009;107(2):126-33.

16. Del Pino M, de Orden A, Arenas A, et al. Argentine references for the assessment of body proportions from birth to 17 years of age. Arch Argent Pediatr. 2017;115(3):23440.

17. Cole TJ, Freeman JV, Preece MA. British 1990 growth reference centiles for weight, height, body mass index and head circumference fitted by maximum penalized likelihood. Stat Med. 1998;17(4):407-29.

18. De Onis M, Onyango AW, Borghi E, et al. Development of a WHO growth reference for school-aged children and adolescents. Bull World Health Organ. 2007;85(9):660-7.

19. WHO multicentre growth reference study group. WHO child growth standards: Length / height for age, weight for age, weight for length, weight for height and body mass index for age: methods and development. Ginebra, World Health Organization, 2006. [Consulta: 1 de julio de 2017]. Disponible en: www.who.int/childgrowth/standards/ Technical_report.pdf.

20. Brook CGD. Clinical Paediatric Endocrinology. 3rd ed. Oxford: Blackwell Science; 1995.

21. Cremin B, Coogman H, Spranger J, et al. Wormian bones in osteogenesis imperfecta and other disorders. Skeletal Radiol. 1982;8(1):35-8.

22. Caffey J, Silverman F. Pediatric X-Ray Diagnosis: a texbook for students and practitioners of pediatrics surgery \& radiology. 5th ed. Chicago: Year Book Medical Publishers; 1967.

23. Caffey J, Madell S. Ossification of the Pubic Bones at Birth. Radiology. 1956;67(3):346-50.

24. Eich GF, Babyn P, Giedion A. Pediatric pelvis: radiographic appearance in various congenital disorders. Radiographics. 1992;12(3):467-84.

25. Golan I, Baumert U, Hrala BP, et al. Dentomaxillofacial variability of cleidocranial dysplasia: clinicoradiological presentation and systematic review. Dentomaxillofac Radiol. 2003;32(6):347-54. 